\title{
Discovery of unusual large group delay in microwave millisecond oscillating events
}

\author{
G. D. Fleishman ${ }^{1,2}$, Q. J. Fu ${ }^{1}$, G.-L. Huang ${ }^{3}$, V. F. Melnikov ${ }^{1,4}$, and M. Wang ${ }^{1}$ \\ 1 Beijing Astronomical Observatory, National Astromonical Observatories, Beijing 100012, PR China \\ e-mail: fuqj@cenpok.net \\ 2 Ioffe Institute for Physics and Technology, 194021 St.Petersburg, Russia \\ 3 Purple Mountain Observatory, National Astromonical Observatories, Nanjing 210008, PR China \\ e-mail: guangli@public1.ptt.js.cn \\ 4 Radiophysical Research Institute, 603950 Nizhny Novgorod, Russia \\ e-mail: meln@nirfi.sci-nnov.ru
}

Received 13 March 2001 / Accepted 17 January 2002

\begin{abstract}
Two events demonstrating periodic narrowband millisecond pulsations of radio emission both in intensity and polarization degree are analyzed. Large time delays between $L$ - and $R$-polarization components are found. The radio emission is shown to be generated as unpolarized by a plasma mechanism at the second harmonic of the upper-hybrid frequency. Observed oscillations of the radiation polarization degree arise due to group delay between extraordinary and ordinary modes along the line of sight. The theoretically predicted dependence of the group delay on frequency $\left(\sim f^{-3}\right)$ agrees excellently with the observed delay frequency dependence. Physical parameters of the emission source and "delay site" are determined within the proposed model.
\end{abstract}

Key words. Sun: radio radiation - Sun: flares - Sun: general

\section{Introduction}

Solar flares are known to produce various types of decimetric and microwave solar radio bursts, both gradual (smoothed) and rapidly fluctuating. The large variety of radio emission types has been challenging for many decades because of the lack of physical classification providing clear discrimination between the different types, self-consistent interpretation and reliable plasma diagnostics.

We emphasize that considerable efforts have been recently made to improve instrumentation and methods of data analysis, to develope radio emission mechanisms and their respective models. As a result, the radio emission measurements and analysis can now be done very carefully providing much deeper and more detailed plasma diagnostics than before.

Decimetric and microwave solar radio bursts frequently produce millisecond fine structures (Slottje 1981; Güdel \& Benz 1988; Allaart et al. 1990; Isliker \& Benz 1994; Jiřička et al. 2001; Fu et al. 2000). Some of them

Send offprint requests to: G. D. Fleishman, e-mail: gregory@sun.ioffe.rssi.ru appear as quasiperiodic pulsations. The short time scale of the pulsations can be naturally interpreted in terms of the pulsating mode of the underlying coherent emission mechanism (Aschwanden 1987; Fleishman \& Melnikov 1998). Loop oscillations usually provide radio pulsations with longer periods $(\tau>1 s$, Aschwanden 1987) and smaller modulation depth and are accompanied by pulsations of the same period in other bands of electromagnetic waves (for example, at mm-wavelengths, hard X-rays).

This paper analyzes events that reveal quasiperiodic millisecond pulsations in both intensity and polarization. While the pulsations with $\tau \sim 50 \mathrm{~ms}$ in the range $f \sim 3 \mathrm{GHz}$ have been recorded many times (Stepanov \& Yurovsky 1990; Isliker \& Benz 1994; Fleishman et al. 1994a), the cases with a time delay between opposite polarizations have not been reported yet.

Actually, a delay between ordinary $(\mathrm{O})$ and extraordinary $(\mathrm{X})$ waves must arise in magnetized plasma due to wave dispersion providing different values of group velocities of $\mathrm{O}$ and $\mathrm{X}$ waves. The effect of radio wave dispersion has been widely used in Astrophysics for cosmic plasma diagnostics. For example, we mention the wellknown investigations of the dispersion measure in the 
interstellar medium from the dispersion of radio pulsar signals (Cordes et al. 1991) and the rotation measure from polarization of galactic and extragalactic objects (Pynzar' 1993).

For the solar case the effect of group delay between waves of opposite polarities has been recently evaluated by Benz \& Pianezzi (1997). The typical group delay has been found to be of the order of tens of ms if (i) the radio emission is generated as partly polarized and (ii) the frequency of the radio emission is close to plasma eigenfrequencies (plasma frequency $\omega_{\text {pe }}$ and gyrofrequency $\omega_{\mathrm{Be}}$ ).

Benz \& Pianezzi (1997) studied the delay between different polarities of partly polarized millisecond radio spikes and found the delay to be of the order of $100 \mu \mathrm{s}$. They concluded that radio spikes are generated in their source as fully polarized while the observed polarization degree arises in a different region in the corona where the plasma frequency and gyrofrquency are much less than the spike frequency.

It is important to note that the delay found in observations may consist of both group delay and other contributions. For radio spikes the total (observed) delay is very small, consequently, the group delay is very small as well. So, the main conclusion of Benz \& Pianezzi (1997) about strong polarization of spikes in their source is correct independent of the bulk contribution to the observed delay - the group effect or other effects.

Unlike to millisecond radio spikes, the millisecond pulsations under study reveal a large total delay. Thus, it is very important to prove if the bulk of the delay is group delay or not. For this purpose we will use the frequency dependence of the observed delay. The expected frequency dependence is easy to find from the general expression

$\Delta t_{\mathrm{g}}=\int_{0}^{\infty}\left(\frac{1}{v_{x}}-\frac{1}{v_{\mathrm{o}}}\right) \mathrm{d} z$

where $v_{x}, v_{\mathrm{o}}$ are the group velocities of extraordinary and ordinary waves respectively, $z$ is the coordinate along the ray path, and $\Delta t_{\mathrm{g}}$ is the group delay between the two magnetoionic modes at the frequency $\omega$. If we substitute here the approximate values of the group velocities valid for $\omega^{2} \gg \omega_{\mathrm{pe}}^{2}, \omega_{\mathrm{Be}}^{2}\left(\omega_{\mathrm{pe}}, \omega_{\mathrm{Be}}\right.$ are the plasma frequency and gyrofrequency of electron):

$\frac{c}{v_{x, \mathrm{o}}} \approx 1+\frac{\omega_{\mathrm{pe}}^{2}}{2 \omega^{2}}-\sigma \frac{\omega_{\mathrm{pe}}^{2} \omega_{\mathrm{Be} \|}}{\omega^{3}}$,

where $c$ is the speed of light and $\omega_{\mathrm{Be} \|}=\omega_{\mathrm{Be}} \sin (\theta), \theta$ is the angle between the line of sight and magnetic field; $\sigma=1$ for O-mode and $\sigma=-1$ for X-mode. Equation (1) together with Eq. (2) provides:

$\Delta t_{\mathrm{g}}=\frac{2}{c} \int_{0}^{\infty} \frac{\omega_{\mathrm{pe}}^{2}(z) \omega_{\mathrm{Be} \|}(z)}{\omega^{3}} \mathrm{~d} z$.

Thus, the group delay is proportional to $f^{-3}$; this prediction is tested and found to be correct for the events in the paper.
Note that the integral (3) can easily be expressed from the rotation measure $R M$ :

$$
\begin{aligned}
R M & =\frac{e^{3}}{2 \pi m_{\mathrm{e}}^{2} c^{4}} \int_{0}^{\infty} n(z) B_{\|}(z) \mathrm{d} z \\
& =2.7 \times 10^{-17} n_{\mathrm{s}} B_{\| \mathrm{s}} H
\end{aligned}
$$

where $n_{\mathrm{s}}, B_{\mid \mathrm{s}}$ are the electron number density and longitudinal component of magnetic field at the source and $H$ is an effective scale of gradual coronal inhomogeneity, $R M$ is in $\mathrm{rad} / \mathrm{cm}^{2}$. We do not assume here any specific laws for variations of $n(z)$ and $B_{\|}(z)$ with the altitude $z$. Further analysis just assumes the physical values to be free from any exotic (e.g., singular) behavior, so the effective scale $H$ is of the same order of magnitude as the real scales of $n$ and $B$ variation in the solar corona.

The organization of the paper is as follows. Section 2 describes the observations. Data analysis (periodicities, frequency dependences etc.) is done in Sect. 3. The interpretation and diagnostics of coronal plasma are done in Sect. 4, where we suggest a model describing observational properties of the events naturally and derive physical paramerers of both the radiation source and the region producing the time delay between $L$ and $R$ polarizations along the line of sight (the delay site). Finally, we discuss the results obtained in Sect. 5 .

\section{Observations}

\subsection{Instrumentation}

The events were recorded with the spectrometer at 2.6-3.8 GHz of the Beijing Astronomical Observatory (Fu et al. 1995). The sampling interval of the instrument is $8 \mathrm{~ms}$ for the full frequency range in two polarization modes ( $R$ and $L$ ). The range is divided into four $300 \mathrm{MHz}$ bands consisting of 30 parallel channels each. A computer takes data from the first to the forth channel in the first half time interval (4 ms) for the left circular polarization, and then, in the second half interval $(4 \mathrm{~ms})$, for the right circular polarization. The first $0.8 \mathrm{~ms}$ of $1 \mathrm{~ms}$ time interval for every $300 \mathrm{MHz}$ band is the integration time, and the last $0.2 \mathrm{~ms}$ is for other tasks (sampling, keeping, etc) for all 30 channels.

\subsection{Overview of the events}

Two narrowband pulsation trains were recorded on the rising phase of a continuum microwave burst on 02 November 1997 at frequencies $f=2.80-2.89 \mathrm{GHz}$ and 3.02-3.09 GHz from 03:02:17 UT to 03:02:21 UT (Figs. 1-2). At the time of the burst an impulsive microwave emission near $3 \mathrm{GHz}$ was also detected at the Hiraiso station, with the maximal flux density of $5 \mathrm{sfu}$, and an intense group of type III bursts was observed in decimetric and metric wavelengths (SGD 1997). GOES detected a short impulsive soft X-ray burst as well. A corresponding $\mathrm{H}_{\alpha}$ flare (SB) occured near the center of the 
solar disc (S20 E01). At the Beijing observatory the microwave burst was observed at noon, when the Sun was high in the sky, so the probability of interference arriving at side lobes was quite low. On other days we did not find any interference similar to the pulsations. All this provides ample evidence for the phenomenon under discussion to be of solar origin.

The dynamic spectra of the pulsations are shown in Fig. 2 for $L$ and $R$ polarizations. In Fig. 3 time profiles of total intensity, $L$ intensity, $R$ intensity, and polarization degree at $f=2.85 \mathrm{GHz}$ are shown. Extended fragments of the curves in Fig. 4 clearly display that for both events the pulsations in opposite polarizations are shifted with respect to each other for a considerable part of the period. Such a delay has never yet been reported for millisecond pulsations of solar radio emission. The distinctive feature of both events (trains) is that both intensities and the polarization degree oscillate with a period of $\tau \approx 40 \mathrm{~ms}$. From Fig. 3 we can note that the record of total intensity seems to be more irregular than that for each of the polarized components $(L$ or $R$ ). The polarization degree reaches values close to $100 \%$. Averaging the records over time does not reveal any significant polarization degree, so the emission is unpolarized on average (the mean value is $1-10 \%$, depending on the integration time).

\section{Data analysis}

\subsection{Observed periodicity and Fourier spectra of the oscillating events.}

To analyze the pulsation events quantitatively we apply the method of Fourier analysis and cross-correlation. Fourier spectra of both high- and low frequency events (see Fig. 2) are shown in Fig. 5. Narrow spectral peaks are well distinquished near $\tau=40 \mathrm{~ms}$ and $\tau=20 \mathrm{~ms}$ for both events. Their magnitudes considerably exceed the noise level. The second (two times less intense) peak is the second harmonic in the Fourier transform of the pulsations. The second peak becomes the largest for the total intensity (Fig. 5, central panels) because here we have two peaks per main period, the first from $R$-waves and the second from $L$-waves. Unlike the lower frequency event, the high-frequency one displays three additional weak spectral peaks at $\tau \approx 29,67$ and $100 \mathrm{~ms}$, which reflects the more irregular temporal behavior of oscillations in the event. Detailed profiles of the main spectral peak for both events are shown in the lower panels of Fig. 5. They are obtained with the method of adding zeros that allows one to improve the precision of spectral peak frequency estimates when using the Fast Fourier Transform (FFT) (Marple, Jr. 1987). The width of the peak at the level of half a magnitude $(F W H M)$ is about $1 \mathrm{~ms}$.

The position of the spectral maximum and, therefore, the period of pulsations, $\tau_{\mathrm{p}}$, varies slightly $(0.2-0.4 \mathrm{~ms})$ depending on the duration of a selected segment of the event, the sense of polarization and the observing frequency (Fig. 6). However, in this case we cannot say that the differences in periods are genuine. Indeed, relatively short durations of the pulsation trains, $\Delta t \sim 2-3 \mathrm{~s}$ for our events, put basic limitations on the error of period determination $\Delta \tau$ :

$\Delta \tau \sim \tau^{2} / \Delta t \sim 0.8 \mathrm{~ms}$

This value agrees well with the $F W H M$ of the main spectral peak, which is about $1 \mathrm{~ms}$ (Fig. 5). The period deviations found in our analysis do not exceed $0.8 \mathrm{~ms}$, so we conclude that the analysis does not reveal any confident period variations.

It is possible to get the shape of the general frequency spectrum of the oscillating events using a Fourier magnitude of pulsations at each observing frequency. Such spectra for both polarizations are displayed in Fig. 7. We can see that the general spectra of $R$ - and $L$-polarized components have well-defined maxima at $2.85 \mathrm{GHz}$ and $2.87 \mathrm{GHz}$ respectively; the first is higher in magnitude than the second one. For both components the spectral bandwidth at half a magnitude is about $40-50 \mathrm{MHz}$, that is about $1.5 \%$ of the observing frequency. The shapes and positions of Fourier spectra for both $L$ - and $R$-components are almost the same for any segment of the pulsation event with a duration of more than $0.5 \mathrm{~s}$, as well as for both of the slightly different periods of $L$ - and $R$-components.

\subsection{Frequency dependence of the observed time delays}

It is easy to estimate from (3) that the difference between the group delay at the lowest $(f=2.81 \mathrm{GHz})$ and the highest $(f=2.89 \mathrm{GHz})$ frequency is about

$\delta t_{\mathrm{g}}=\Delta t_{\mathrm{g}}(2.81 \mathrm{GHz}) \cdot 3 \frac{\Delta f}{f_{\mathrm{o}}} \approx 2 \mathrm{~ms}$

for $\delta t_{\mathrm{g}}(2.81 \mathrm{GHz}) \approx 20 \mathrm{~ms}, \Delta f \approx 80 \mathrm{MHz}, f_{\mathrm{o}}=$ $2.81 \mathrm{GHz}$.

The available sampling interval of the spectrometer, $8 \mathrm{~ms}$, does not allow us to check directly the prediction of the model. However, it is well known (see, e.g., Max 1981) that for quasiperiodic oscillations with a narrow spectral peak (Fig. 5) the Fourier method of precise phase determination can be applied. This method has widely been used in interferometry (Thompson 1990). According to the method, a digitized signal carries quite precise information on parameters of a quasiperiodic real signal, including the phase, if the sampling rate provides more than two data points per period of the signal (Nyquist theorem). For our case this condition is fulfilled perfectly: we have 5 data points per period. Thus, we expect to obtain the precision of delay estimates sufficient to verify the theoretical predictions. 


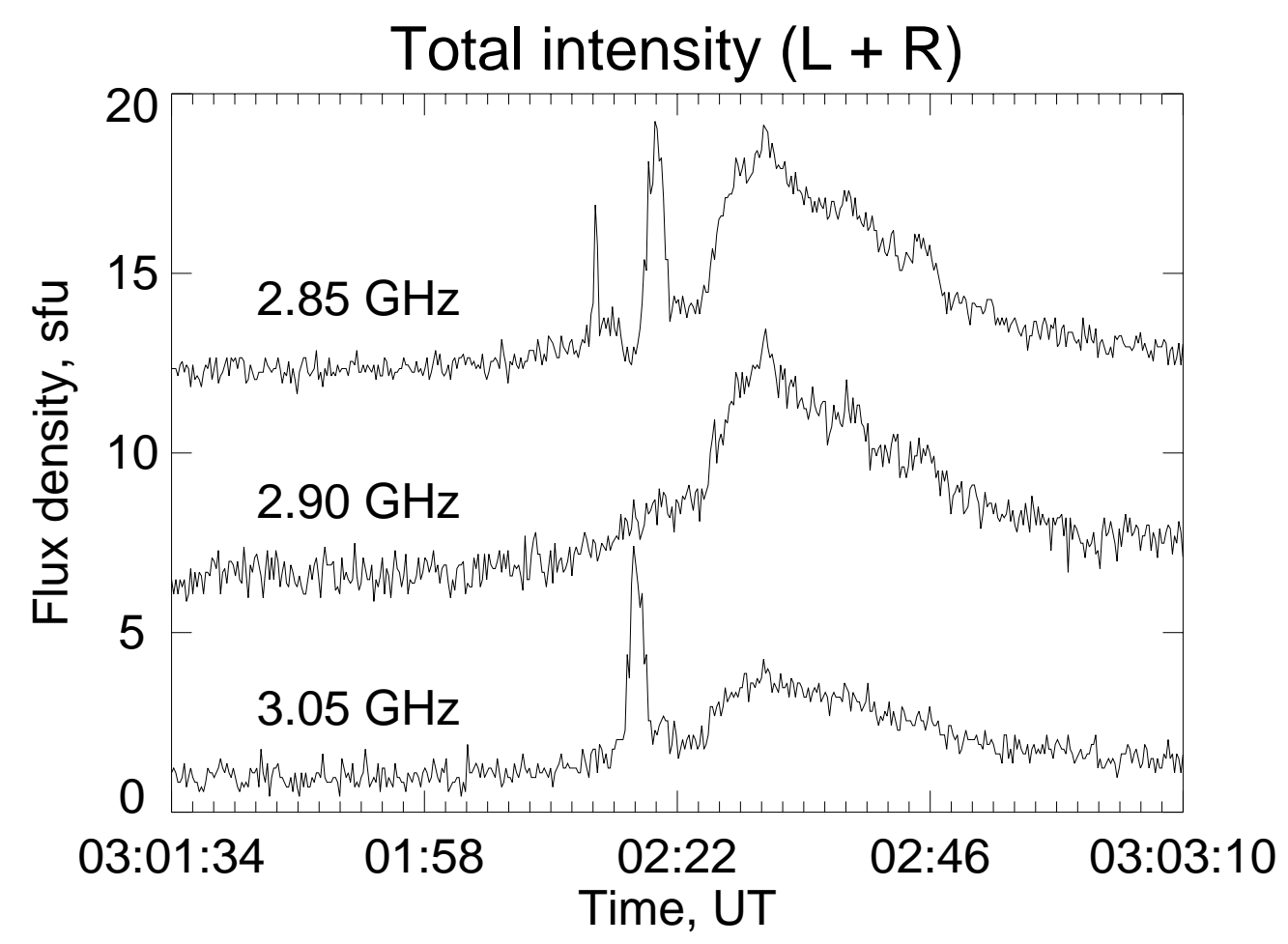

Fig. 1. Time profiles integrated over $200 \mathrm{~ms}$ of the microvawe burst on 2 November 1997. The narrowband pulsation events occured on the rise phase of a continuum microwave burst during the time 03:02:17 UT to 03:02:21 UT.

\subsubsection{Fourier method of delay determination}

The Fourier analysis allows us to calculate the phase of each of the frequency components of the complex signal spectrum $I(\nu)$ using the following expression (Max 1981):

$\phi(\nu)=\arctan \left(\frac{\operatorname{Im}[I(\nu)]}{\operatorname{Re}[I(\nu)]}\right)$,

where $\operatorname{Re}[I(\nu)]$ and $\operatorname{Im}[I(\nu)]$ are the real and imaginary parts of the Fourier transform at the frequency $\nu$. For quasiperiodic signals like the narrowband oscillations under analysis, the problem is to find the phase of the signal at the frequency of the spectral peak $\nu_{\mathrm{p}}=1 / \tau_{\mathrm{p}}$ (Fig. 5). After calculating the phases of $R$ and $L$ polarized oscillations in a given time segment, we can get a time delay between the two signals as follows:

$\Delta t_{\mathrm{d}}=\frac{\phi_{R}\left(\nu_{\mathrm{p}}\right)-\phi_{L}\left(\nu_{\mathrm{p}}\right)}{2 \pi \nu_{\mathrm{p}}}$

where $\nu_{\mathrm{p}}=\nu_{\mathrm{p} L}$ or $\nu_{\mathrm{p}}=\nu_{\mathrm{p} R}$ is one of the two values of the slightly different peak frequencies calculated separately for $R$ and $L$ polarized oscillations respectively (see Fig. 6). The further analysis uses both values.

Consider a simple case when real signals have only one true delay. The physical delay $\Delta t_{\mathrm{ph}}$ can be either less than value of the pulsation period, $\tau$, or larger than it, either positive or negative. How should the unique delay manifest itself in the Fourier analysis applied? It is important to understand that even if the physical delay is larger than the period,

$\Delta t_{\mathrm{ph}}>\tau$, we will detect a delay, $\Delta t_{\mathrm{d}}$, which belongs to the interval

$0<\left|\Delta t_{\mathrm{d}}\right|<\tau$,

so that

$\Delta t_{\mathrm{ph}}=\Delta t_{\mathrm{d}}+m \tau$

where $m$ is an integer number (positive or negative). Moreover, we cannot distinguish between the values $\Delta t_{\mathrm{d}}$ and $\tau-\Delta t_{\mathrm{d}}$; both of them belong to the interval (10).

So, we obtain a series of values, while we know that one of them only is true. To specify it we should invoke some additional ideas. We discuss this later. However, we could expect a priori that the number $m$ cannot be large. Actually, for $m=1$ we have $\Delta t_{\mathrm{ph}} \approx 60 \mathrm{~ms}$, for $m=2$ we have $\Delta t_{\mathrm{ph}} \approx 100 \mathrm{~ms}$ etc. These values (if being typical for pulsations) must have been found long ago by instruments with a time resolution lower than $8 \mathrm{~ms}$.

\subsubsection{Delay errors}

The calculated delay values are found to vary considerably for different lengths of chosen time segments as well as for different observable frequencies (Fig. 8). For very short segments and for channels with weak signals the value of the delay becomes too unstable. For the low frequency pulsation event the stable values could be obtained only for durations of segments of more than $0.5 \mathrm{~s}$ and for frequencies in the range of $2.81-2.89 \mathrm{GHz}$ where the signal is quite strong (see Fig. 7). It is clear that before going on we have to define what influences the stability of the delay determination and how to estimate its possible error. 

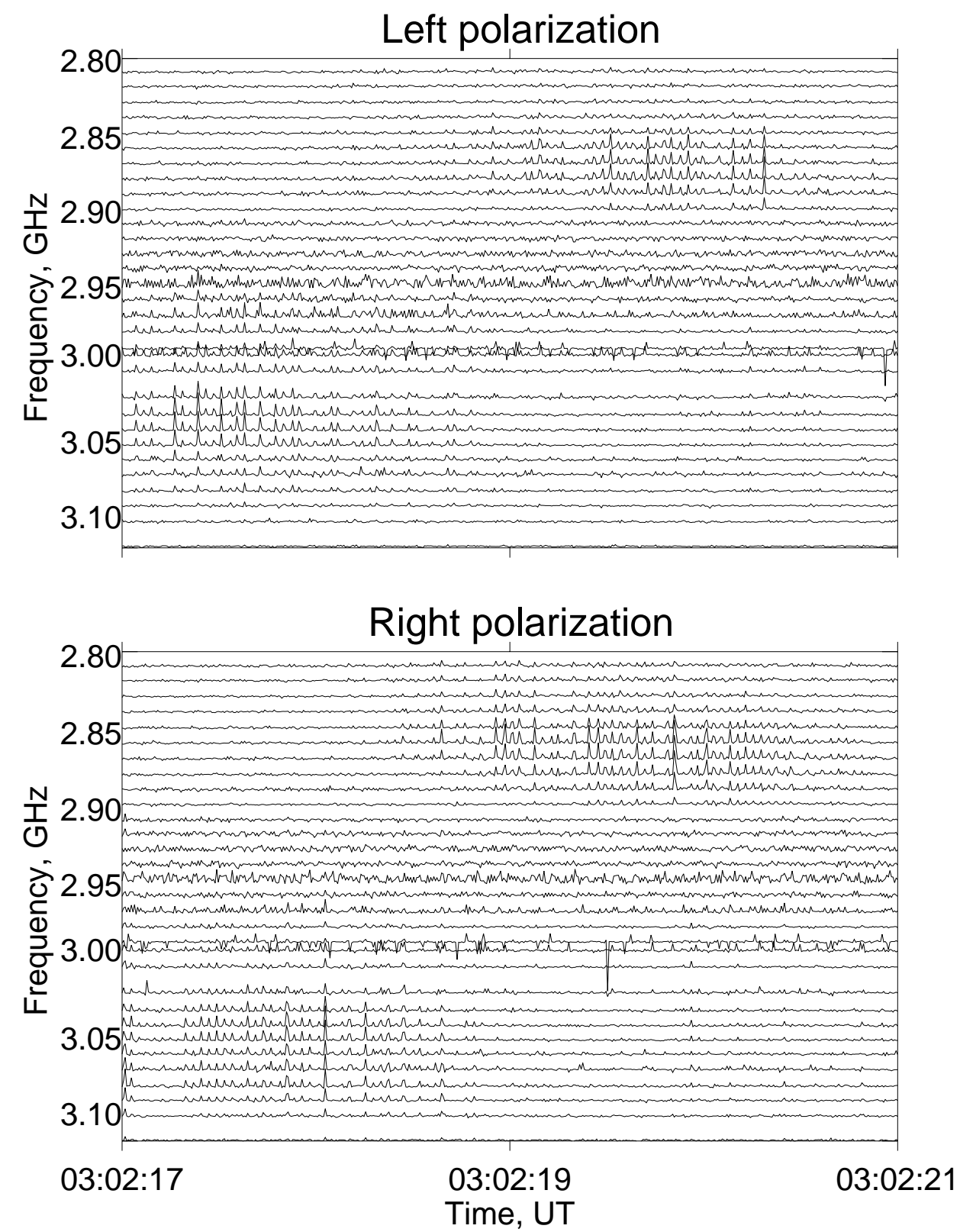

Fig. 2. Dynamic spectra of the pulsations for $L$ and $R$ polarizations. Two narrow-band pulsation events are clearly seen around $2.85 \mathrm{GHz}$ and $3.05 \mathrm{GHz}$. At these frequencies the pulsation signal-to-noise ratio achieves values of $5-7$ during the events. Some channels (around 2.95 and $3.00 \mathrm{GHz}$ ) are noisy.

The phase error for an oscillating function, with some noise superimposed on it, is defined as follows (Thompson et al. 1990):

$\delta \phi=\frac{\text { noise }}{\text { signal }} \frac{1}{\sqrt{K}}$

where $K$ is the total number of periods along the oscillating function.

To get an estimate of the error in the time delay between $R$ and $L$ quasiperiodic components we use the obvious relation $\delta t_{\mathrm{d}}=\delta \phi /\left(2 \pi \nu_{\mathrm{p}}\right)$ and obtain the following expression:

$\delta t_{\mathrm{d}}=\frac{\sigma_{\mathrm{N}}}{\sqrt{2} \sigma_{\mathrm{S}}} \frac{1}{\left(\sqrt{K} 2 \pi \nu_{\mathrm{p}}\right)}$ where $\sigma_{\mathrm{N}}$ is the standard deviation of a noise signal before the beginning of pulsations or after the end of them; $\sigma_{\mathrm{S}}$ is standard deviation of the signal during the pulsations.

So, the delay error depends strongly on the specific signal-to-noise ratio and on the number of periods of oscillations. For instance, the delay error is $0.3 \mathrm{~ms}$ for the second, low frequency, pulsation event at $f=2.85 \mathrm{GHz}$ during the first 50 periods where the signal-to-noise ratio is $\sim 5$. The error increases up to $0.8 \mathrm{~ms}$ for the same time interval at lower frequency, $2.81 \mathrm{GHz}$, because of a lower signal-to-noise ratio.

As mentioned in Sect. 2, the artificial $4 \mathrm{~ms}$ delay in sampling the $L$-polarized signal with respect to the 

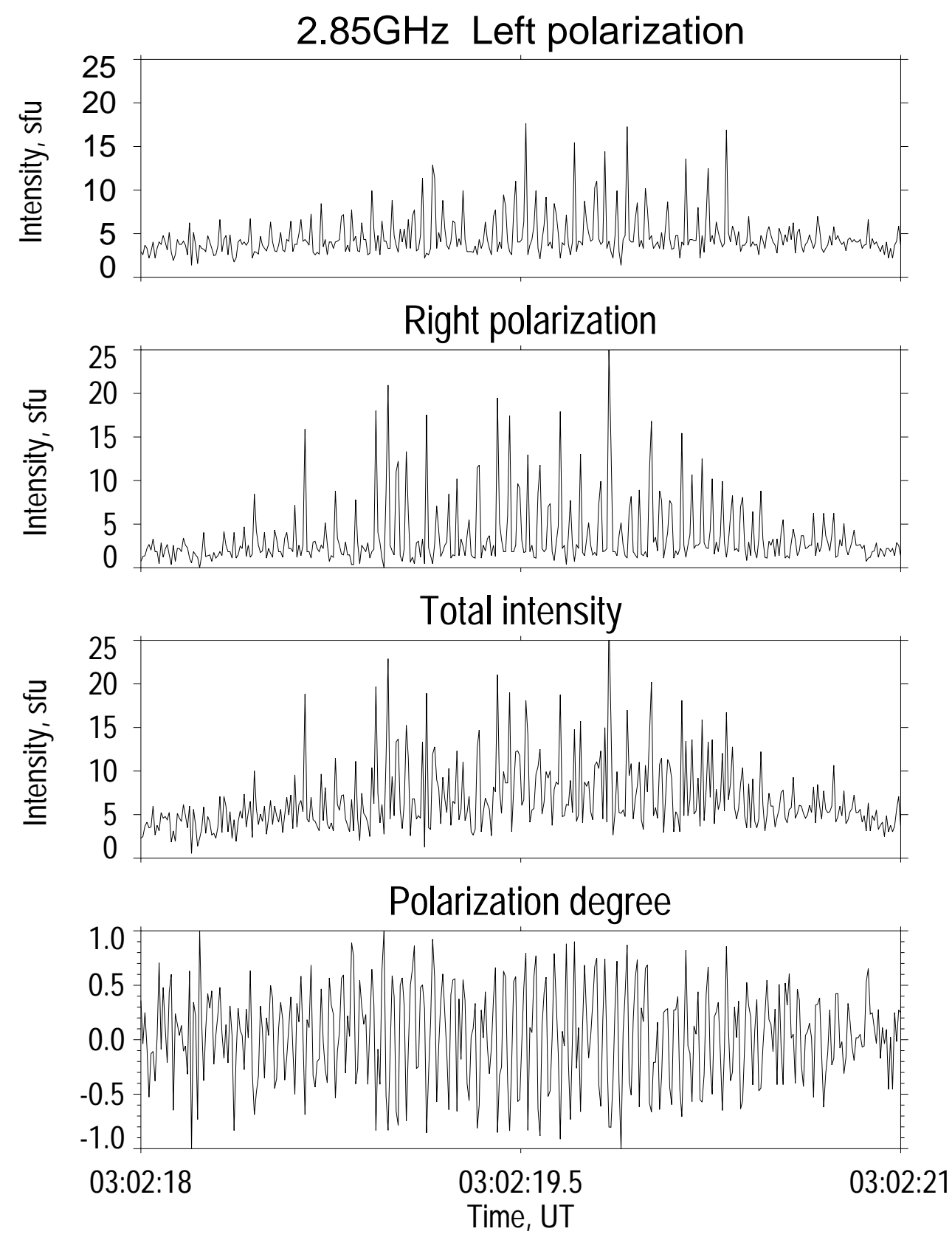

Fig. 3. Time profiles of $L$ intensity, $R$ intensity, total intensity, and polarization degree at $f=2.85 \mathrm{GHz}$. The total intensity curve looks more irregular than the other curves.

$R$-polarized signal at every frequency inside the band 2.60-2.90 $\mathrm{MHz}$ arises systematically due to the sampling procedure. So, to get real delays between $R$ and $L$ components of pulsations, we made a correction for this $4 \mathrm{~ms}$.

\subsubsection{Delay-frequency dependence}

We analyzed different time segments of the oscillation records for both oscillating events and found quite stable negative frequency dependence of the delays with the shape of a power law. The most reliable dependence has been found for the second (low frequency) oscillating event, which is characterized by a single strong Fourier spectral peak. For this event the dependence shape is the power law with the exponent $-(2.8 \pm 0.8)$. The slope varies at the limits depending on the start time and duration of a chosen (restricted) time segment.

A typical plot of the frequency dependence of the delays for one of the $1 \mathrm{~s}$ duration segments being analyzed is shown in Fig. 9. The delays are taken at the frequencies of the Fourier spectral maxima for $R$ (asterisk) and $L$ (triangle) components. As is seen from the plot, the frequency dependence of the delay is very prominent. Normally, for various $1 \mathrm{~s}$ time segments, correlation coefficients vary in the range of $0.80-0.96$. 

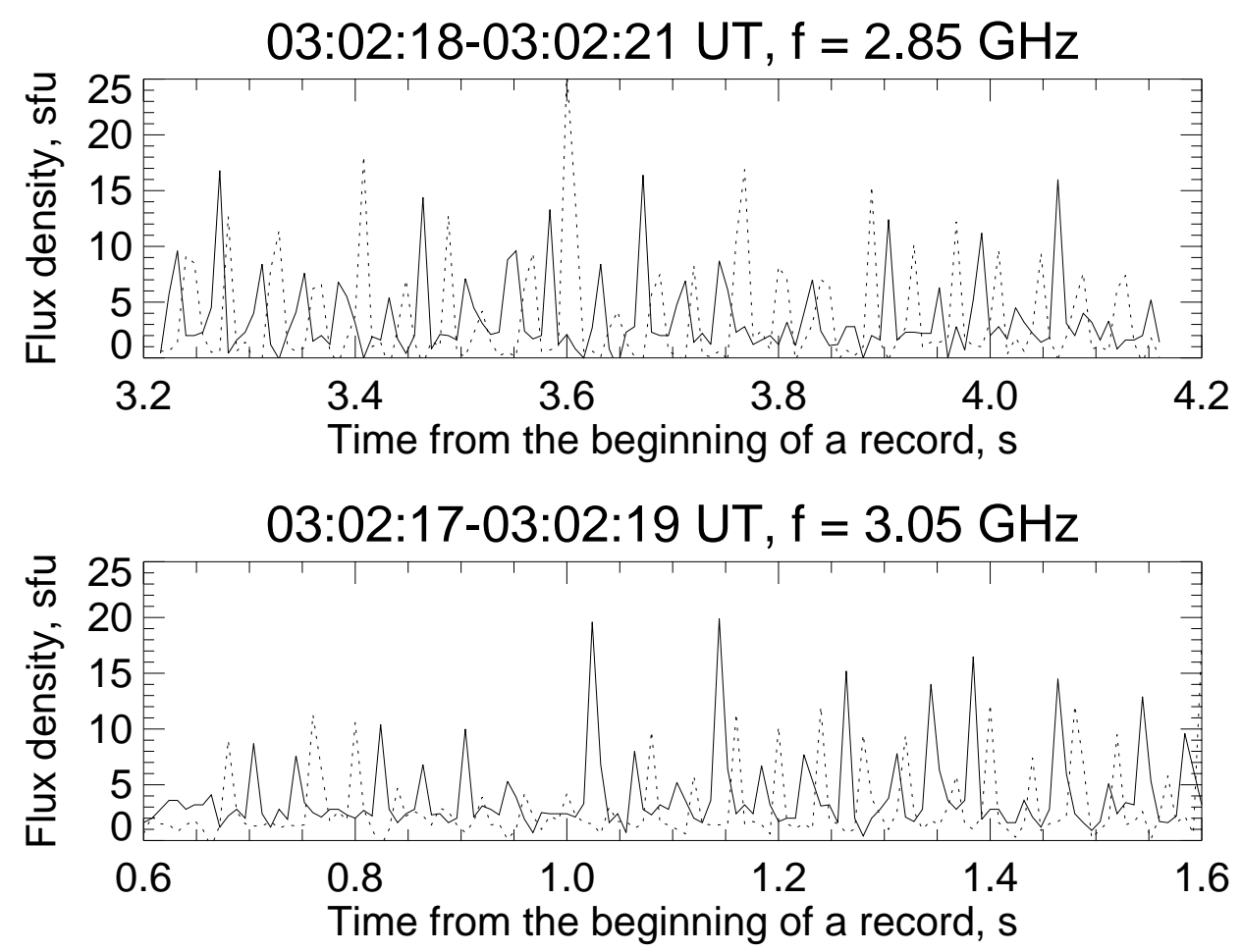

Fig. 4. Selected time segments of the pulsation events at $f=2.85 \mathrm{GHz}$ and $f=3.05 \mathrm{GHz}$ during a 1 s time segment in $R$ (solid line) and $L$ (dotted line) polarizations. A time shift between components is clearly visible.

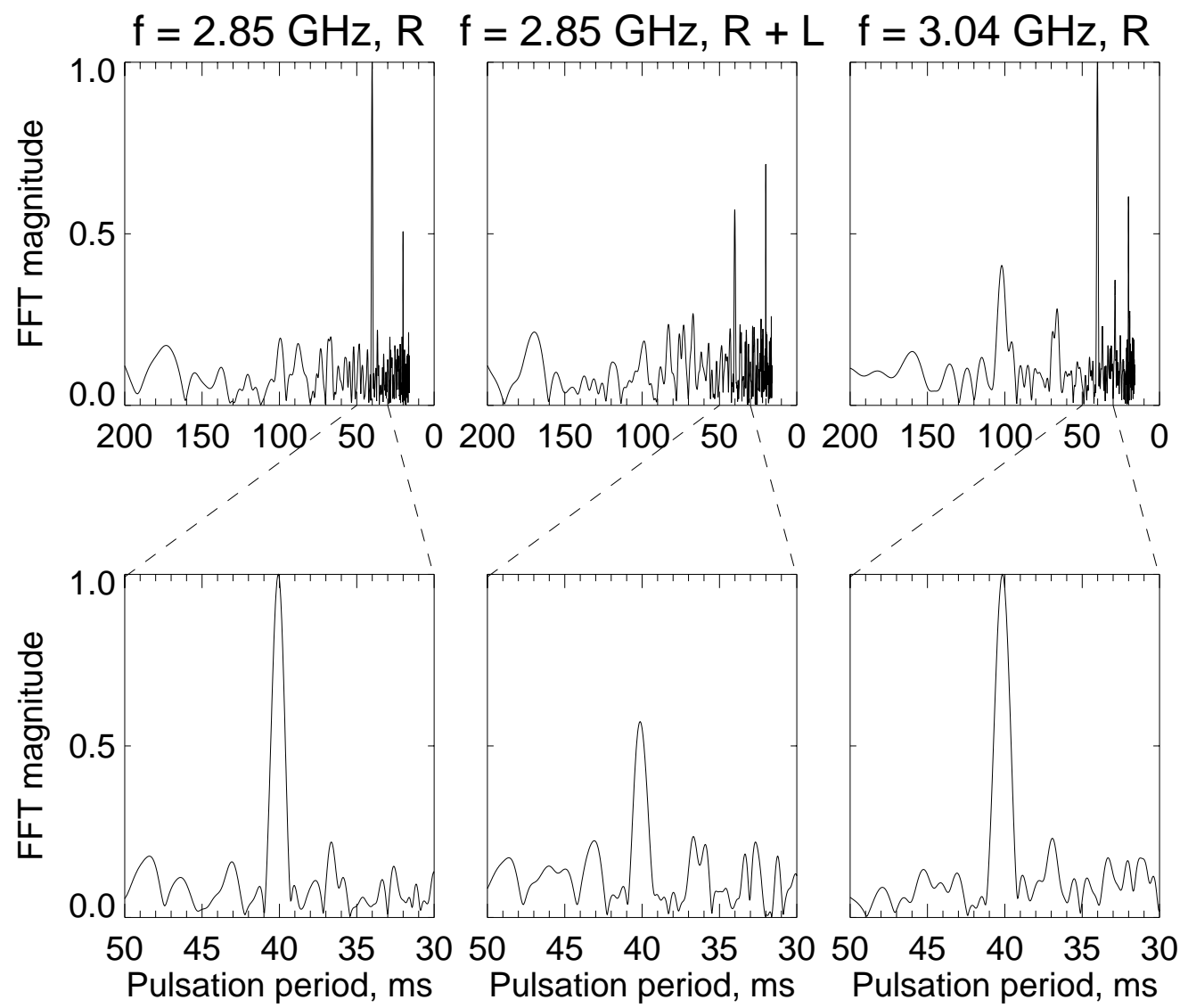

Fig. 5. Normalized Fourier spectra (periodograms) of the $R$-polarized component (left panels) and the total intensity (central pannels) for the lower frequency event and $R$-polarized component of higher frequency event (right panels) obtained for the time intervals of 03:02:18.5-03:02:20.5 UT and 03:02:17.0-03:02:18.5 UT (see Fig. 2), respectively. The bottom panels display extended Fourier spectra near the corresponding main spectral peaks. 


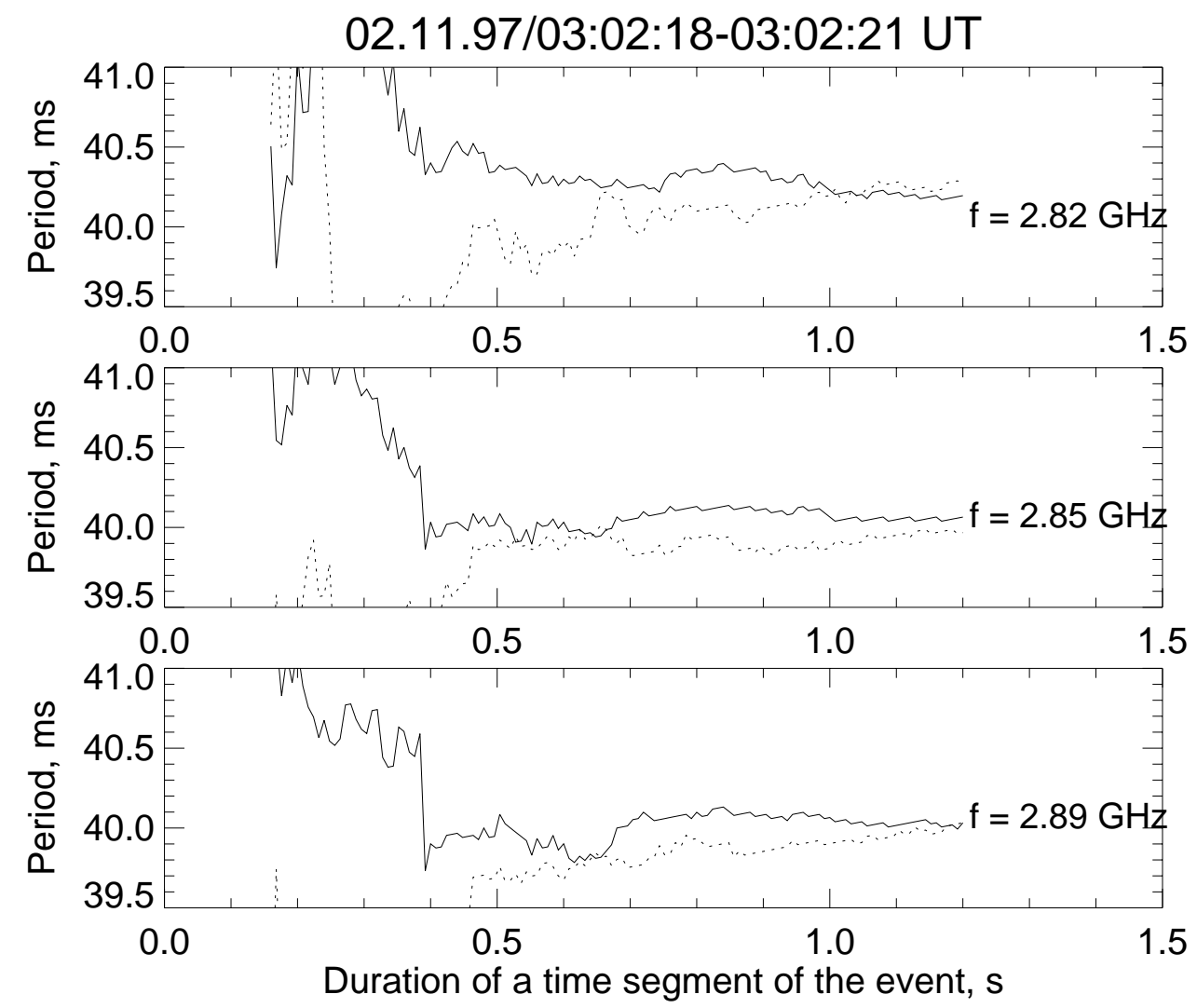

Fig. 6. Dependence of the pulsation period $\tau_{\mathrm{p}}$ at different observing frequencies on the duration of a time segment for $R$ (solid line) and $L$ (dotted line) components.

The correlation plot in Fig. 9 is compatible with the theoretical prediction $\Delta t_{\mathrm{g}} \propto f^{-3}$. The calculated linear regression $\lg ($ Delay $)=a+b \cdot \lg$ (Frequency) gives, with a $95 \%$ probability, $b=-2.99 \pm 0.89$ and the correlation coefficient $r=0.95$ for the points taken at $L$-component Fourier maxima, and, respectively, $b=-3.35 \pm 0.68$ and $r=0.94$ for the points taken at $R$-component Fourier maxima. These numbers are compatible with the averaged $b$ value for the whole event given above.

Note that the differences between delays taken at the pulsation frequencies of spectral maxima for $R$ and $L$ polarized components are quite small, normally inside the error bars. This demonstrates the stability of the delay determination method. Larger error bars at low frequencies reflect the weaker signal-to-noise ratio at these frequencies. This ratio varies with frequency considerably for $R$ $(L-)$ polarization mode: from $1.8(3.1)$ at $2.81 \mathrm{GHz}$ to 6.3 (7.5) at the peaks of the spectra (see Fig. 7) and 3.8 (3.1) at $2.89 \mathrm{GHz}$. In general, the full size of the error bars, $0.5-1 \mathrm{~ms}$, is remarkably smaller than the dynamic range of the delay change, $2 \mathrm{~ms}$, related to the frequency change across the band of the pulsation event.

Figure 9 displays the values of the delay from Eq. (11) for $m=0$. To confirm this choice we used cross-correlation analysis of the records. Indeed, the cross-correlation function of two oscillating signals displays oscillating behavior with local peaks separated by an integer number of periods, which corresponds to the series (11). However, any real oscillating signal has an envelope. Such envelopes must correlate for two correlated signals as well as the oscillations themselves. So we concentrate now on this aspect of the cross-correlation.

For this purpose we can neglect the small differences of the delay at different frequencies and study the $L$ and $R$ signals integrated over all frequency channels. This integration improves the signal-to-noise ratio.

Figure 10 (top) displays the peaks of the crosscorrelation function for the integrated signals as well as the respective Gaussian fit. The maximum of the fit ( $\Delta t=-26 \mathrm{~ms}$, the sign "-" means that $R$ polarized waves arrive first) is marked by the arrow. Figure 10 (bottom) displays the correlation function and the fit for a restricted lag region.

To study the stability of the results we (i)varied the number of integrated channels, (ii)added additional noise into the records, (iii)selected different time segments of the records, and found the delay to be

$\Delta t=-20 \pm 15 \mathrm{~ms}$.

Obviously, this value is compatible with our choice $m=0$, so the actual delay equals the found delay

$\Delta t_{\mathrm{ph}}=\Delta t_{\mathrm{d}} \approx-20 \mathrm{~ms}$.

We omit the sign "-" for convinience and will keep in mind that $R$ polarized waves arrive before $L$ polarized waves. 


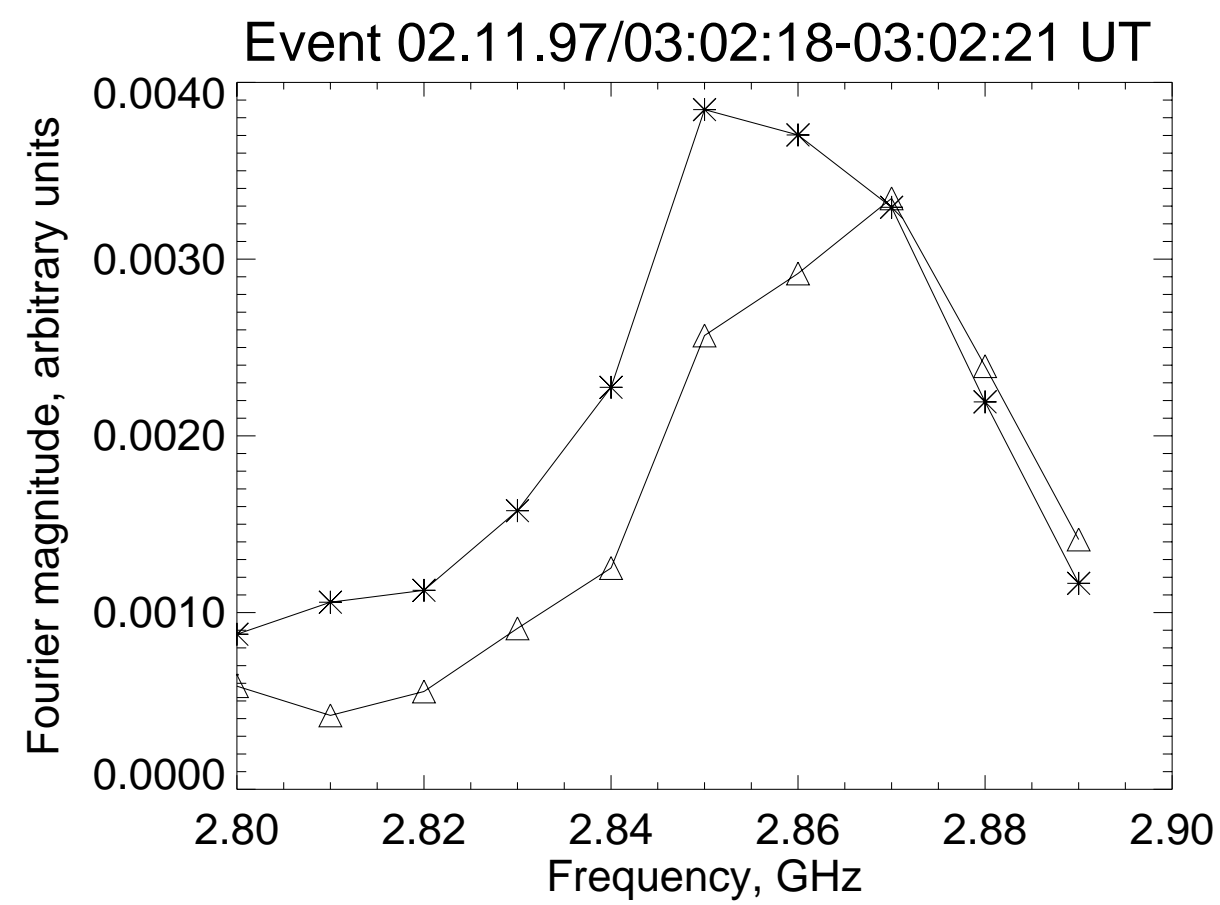

Fig. 7. Dependence of the Fourier magnitude of pulsations on frequency for $R$ (asterisk) and $L$ (triangle) polarized components.

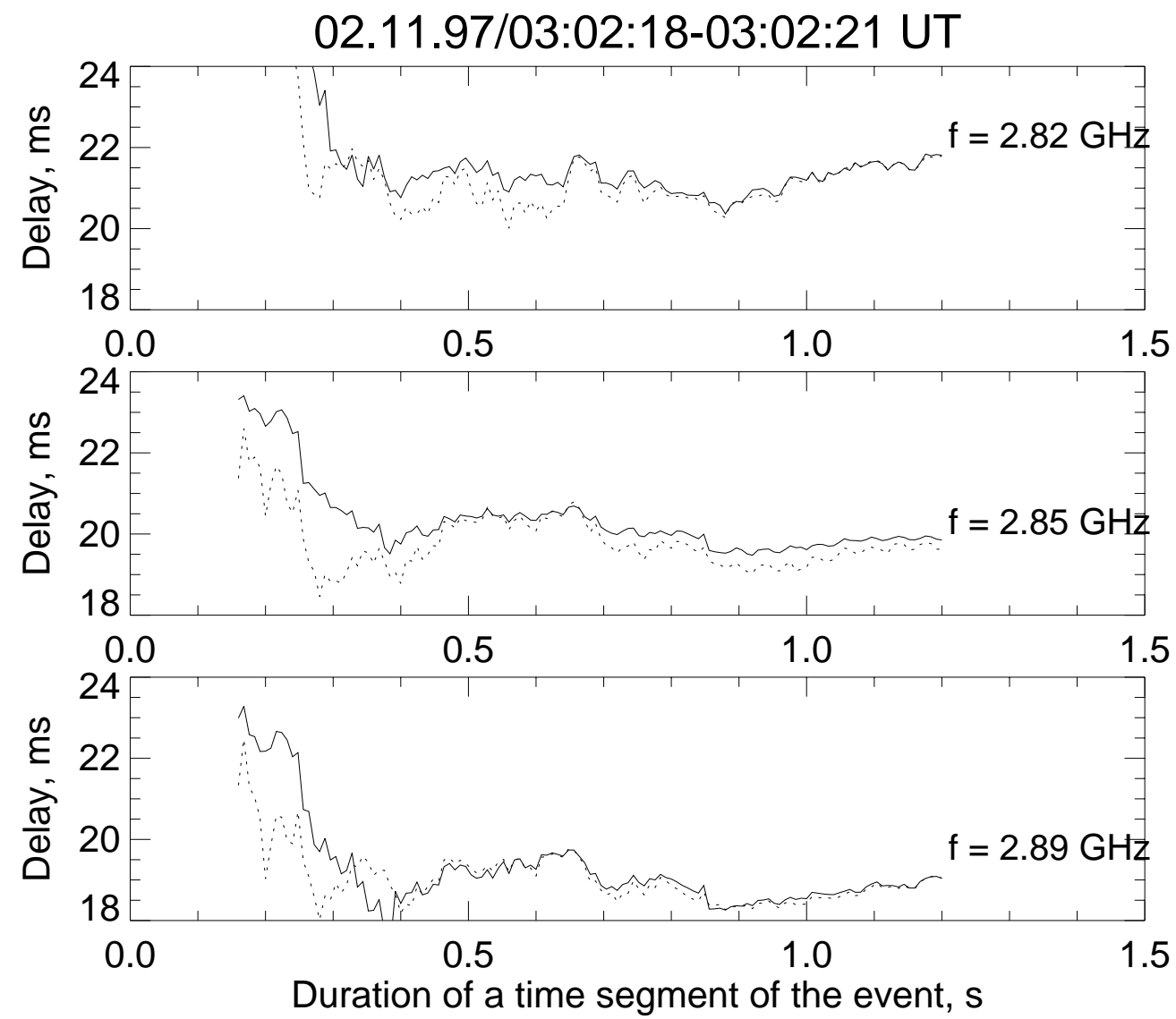

Fig. 8. Delay variations at different observing frequencies depending on the duration of oscillation time segments. Variations are shown separately for delays defined at the peak frequencies of $R$-polarized (solid line) and $L$-polarized (dots) signals. 


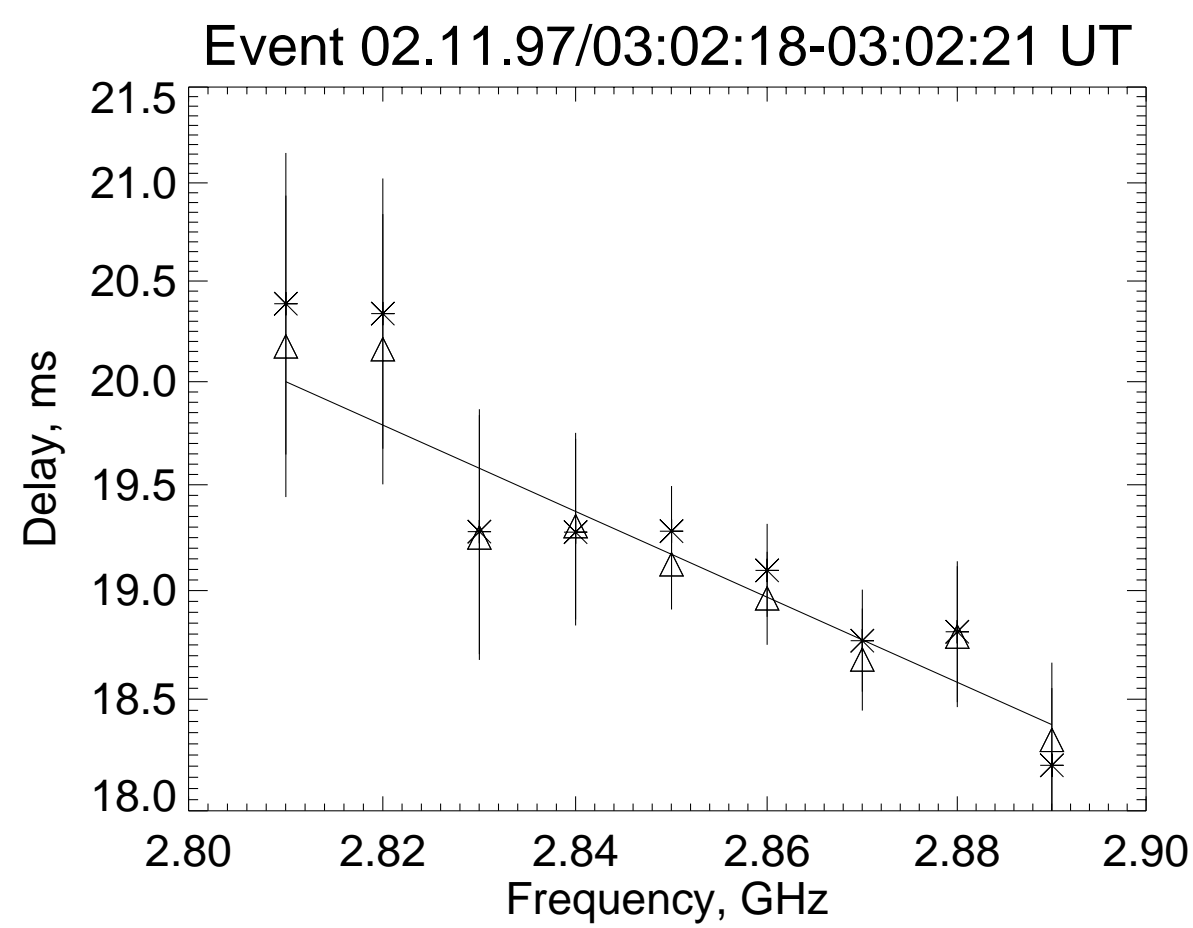

Fig. 9. Frequency dependence of the delay between $R$ - and $L$-polarized components of oscillations on a log-log scale. The delays are taken at the frequencies of Fourier maxima for $R$ (asterisk) and $L$ (triangle) components. The solid line is the theoretical prediction for the group delay effect, $\propto f^{-3}$.

\section{Physical model and plasma diagnostics}

\subsection{Emission mechanism}

Let us discuss the emission mechanism that could generate the pulsations under study. Aschwanden (1987) discusses a lot of different possibilities providing pulsations of solar radio emission based on both coherent and incoherent mechanisms. The analyzed pulsations have a very narrow spectral bandwidth, which requires for a coherent mechanism to be involved. Oscillations of coherent radio emission can be produced either when periodic injection of fast electrons into the radio source occures or when underlying instability operates in a oscillatory regime. The first possibility can obviously be excluded because no similarity with type III or IIIdm bursts is found.

There are two coherent mechanisms producing pulsating radio emission, electron cyclotron maser emission (Aschwanden \& Benz 1988) and nonlinear plasma mechanism (Zaitsev \& Stepanov 1983). To specify which of the mechanisms is responsible for our events, let us consider polarization of the pulsations.

The observed polarization degree displays quasiperiodic oscillations. In Section 3, the oscillating behaviour of the polarization degree has been shown to result from the shift between $L$ and $R$ records connected with group delay between $\mathrm{X}$ and $\mathrm{O}$ modes in the corona. This means that the radio emission is generated as weakly polarized in the radio source.

Electron cyclotron maser emission at any harmonic as well as fundamental plasma emission produces strongly (100\%) polarized radio emission, while plasma emission at the second harmonic is moderately or weakly polarized. Thus, we conclude that the pulsations are generated by a plasma mechanism at the second harmonic of the plasma frequency (to be more correct, at the second harmonic of the upper-hybrid frequency).

We should emphasize that the conclusion is the direct consequence of the observed large group delay between two magnetoionic modes. It is important as well that the production of oscillating emission patterns is an intrinsic property of the plasma mechanism, so we do not need any additional assumption to explain the oscillations themselves.

The plasma mechanism of radio emission includes two steps. The first is excitation of plasma (Langmuir, upperhybrid, lower-hybrid etc.) waves by unstable fast electron distribution (say, beam-like or loss-cone). The second one is transformation of the plasma waves into radio waves (ordinary or/and extraordinary). If nonlinear wave-wave interactions are important during the first step, we have a nonlinear plasma mechanism of radio emission (Zaitsev 1971; Zaitsev \& Stepanov 1983).

Numerical study of the nonlinear plasma mechanism of radio emission (Korsakov \& Fleishman 1998; Fleishman \& Korsakov 1999) shows that the energy density of excited plasma (upper-hybrid) waves displays either irregular pulsations or quasiperiodic oscillations depending on the shape and scale of the initial instability region provided by fast electron distribution. Actually, quasiperiodic solutions arise for narrow instability regions, irregular pulsations appear for broader instability regions, and transitions from one regime to the other are possible. 

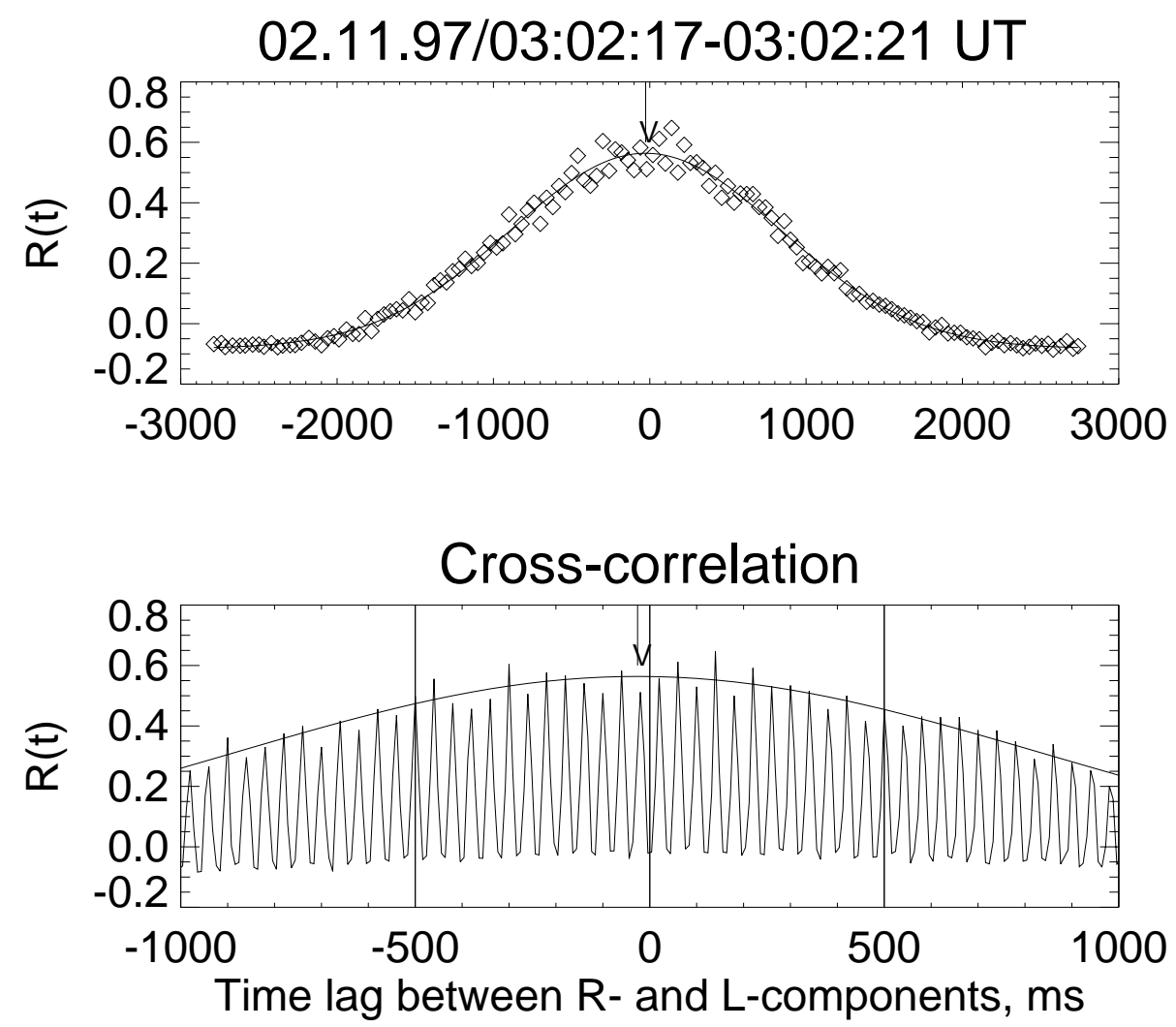

Fig. 10. Cross-correlation function $R(t)$ for the pulsations integrated over nine frequency channels. Top: squares are the local peaks of the cross-correlation function, the solid line is the Gaussian fit. The arrow shows the maximum of the fit. Bottom: the cross-correlation function and the Gaussian fit for a restricted lag region.

To apply the mechanism to the solar case we should take into account that the effective growth rate of plasma waves is the difference between the growth rate provided by unstable fast particles and the collisional and the collisionless damping rates provided by thermal plasma. A narrow instability region (producing quasiperiodic oscillations) can arise if the instability operates slightly above the threshold (defined by the damping rate). In this case we could expect that the instability conditions are fulfilled in some restricted region of nonuniform magnetic loop, while in most of the loop the damping rate exceeds the growth rate, which prevents the generation of plasma waves. Probably, this is the reason for the very small spectral bandwidth of the pulsations under study.

The modulation depth is very high (close to 100\%) for each polarization. This means that the group delay inside the source of radiation is negligible: in the case of considerable group delay inside the source, the record of the corresponding X-mode should be smoothed out and have a smaller modulation depth. Therefore, we conclude that the source size should be small enough,

$L_{\mathrm{s}} \ll c \tau \approx 10^{9} \mathrm{~cm}$

where $\tau$ is the period of oscillations.

\subsection{Plasma diagnostics}

Let us apply the theory of nonlinear plasma mechanism for the pulsations to extract the physical parameters of the radio source from observations.

The upper hybrid (UH) frequency is defined by the dispersion relation

$$
\begin{aligned}
& \omega_{\mathrm{UH}}^{2}=\frac{1}{2}\left(1+3 k^{2} d_{\mathrm{e}}^{2}\right) \\
& \times\left\{\left(\omega_{\mathrm{pe}}^{2}+\omega_{\mathrm{Be}}^{2}\right)+\left[\left(\omega_{\mathrm{pe}}^{2}+\omega_{\mathrm{Be}}^{2}\right)^{2}-4 \omega_{\mathrm{pe}}^{2} \omega_{\mathrm{Be}}^{2} \cos ^{2} \theta\right]^{1 / 2}\right\}
\end{aligned}
$$

and it depends on the angle between the respective wave vector $\boldsymbol{k}$ and magnetic field $\boldsymbol{B}$ and on the wave vector itself; $d_{\mathrm{e}}$ is the electron Debye radius. Loss-cone distribution of fast electrons produces UH-waves in quasitransverse directions (Korsakov \& Fleishman 1998; Fleishman \& Korsakov 1999),

$\omega_{\mathrm{UH}} \approx\left(\omega_{\mathrm{pe}}^{2}+\omega_{\mathrm{Be}}^{2}\right)^{1 / 2}$

so the radio emission frequency $\omega$ is close to $2 \omega_{\mathrm{UH}}$

$\omega \approx 2 \omega_{\mathrm{UH}}$.

Depending on the ratio $Y=\omega_{\mathrm{pe}} / \omega_{\mathrm{Be}}(Y \geq 1)$ the $\mathrm{UH}$ frequency varies from $\omega_{\text {pe }}$ (if $Y \gg 1$ ) to $\sqrt{2} \omega_{\text {pe }}$ (if $Y=1$ ). Actually, we would expect $\omega_{\mathrm{pe}} \approx \omega_{\mathrm{Be}}(Y \approx 1)$ to provide 
the observed time delay between opposite polarizations. Furthermore, extraordinary waves are known to have large cyclotron absorption if $\omega \leq 3 \omega_{\mathrm{Be}}$. Because the averaged polarization degree is very small we must conclude that $\omega_{\mathrm{Be}} \leq \omega / 3$ in the source and along the line of sight. This requirement (together with Eqs. (18), (19)) yields:

$\omega_{\mathrm{Be}} \leq 0.89 \omega_{\mathrm{pe}}$

Taking into account that for the low frequency event

$\omega \approx 2 \pi(2.85 \pm 0.04) \times 10^{9} \mathrm{c}^{-1}$

and $(18,19,20)$, we find the lower limit of the electron number density in the source:

$n \geq 1.3 \times 10^{10} \mathrm{~cm}^{-3}$.

The upper limit can be found if we accept $Y \gg 1$ (which is however incompatible with the large shift observed):

$n<2.5 \times 10^{10} \mathrm{~cm}^{-3}$

in the source.

Furthermore, these equations provide us with the upper level of the magnetic field

$B<325 \mathrm{G}$

in the source and along the line of sight.

As it is shown below, at the frequencies closed to twice upper-hybrid frequency the $\mathrm{X}$ and $\mathrm{O}$ waves have the largest difference of group speeds if

$Y=\sqrt{2} \approx 1.4$.

The observed time shift between the oppositely polarized waves is rather large. Thus, we suppose the physical conditions to be preferable for the effect to be observed, so that $Y \approx 1.5$. The assumption gives rise to the estimates (with an accuracy better than $30 \%$ ):

$n \approx 1.7 \times 10^{10} \mathrm{~cm}^{-3}$

$B \approx 290 \mathrm{G}$

in the source.

The bandwidth of the emission consists from natural bandwidth and broadening due to source inhomogeneity. Obviously, the theoretically predicted natural bandwidth of generated plasma waves should not exceed the bandwidth of the observed pulsations. If the upper-hybrid waves were distributed isotropically, the respective natural bandwidth (under the condition $Y \approx 1.5$ ) were

$\frac{\Delta \omega}{\omega} \sim 10-20 \%$,

while the observed full bandwidth is $\sim 3 \%$.

The model developed in (Korsakov \& Fleishman 1998; Fleishman \& Korsakov 1999) calculates the characteristic values of the angle $\theta$ and wave-vector $\boldsymbol{k}$ of generated upper-hybrid waves and the respective scatter $\Delta k$ and $\Delta \theta$ :

$k d_{\mathrm{e}} \sim 0.1$ $\theta \sim 80^{\circ}$

$\Delta k d_{\mathrm{e}} \sim 0.1$

$\Delta \theta \leq 30^{\circ}$

so $\Delta k \sim k$, and provides the natural bandwidth

$\frac{\Delta \omega}{\omega} \sim 1-2 \%$.

This could mean that the source inhomogeneity contribution into observed bandwidth is comparable with (or less than) the natural bandwidth of the emission mechanism.

Numerical and analytical study of the nonlinear plasma mechanism of radio emission shows that the period of plasma wave oscillations is defined by their effective damping rate:

$\tau=\ln \left(W_{\max } / W_{0}\right) / \gamma_{\text {eff }}$

for large oscillations (Zaitsev 1971; Zaitsev \& Stepanov 1983; Korsakov \& Fleishman 1998; Fleishman \& Korsakov 1999), where $W_{0}$ and $W_{\max }$ are the initial and maximal levels of plasma wave energy density. The effective damping rate can be provided by either thermal plasma or fast electrons themselves. In the first case it equals the collisional damping rate, in the second case it is close to the maximum growth rate. In our case the period of the pulsations is very stable, so we can conclude that the collisional damping rate dominates. On the other hand, the maximum growth rate and the damping rate should be comparable in value, $\gamma_{\max } \sim \gamma_{\text {eff }}$. Indeed, if the growth rate were much larger than the damping rate, the upperhybrid waves would be excited in a more extanded region of the loop providing a larger spectral bandwidth of the radio emission than observed.

Using the found value of period $\tau$, Eqs. $(26,34)$, and the collisional damping rate

$\gamma_{\mathrm{eff}} \approx \nu_{\mathrm{ei}} \approx 60 n T^{-3 / 2}$

we obtain the electron temperature in the source

$T \approx 3 \times 10^{6} \mathrm{~K}$.

Accordingly, we can estimate the maximum growth rate of the upper-hybrid waves as

$\gamma_{\max } \approx(2-3) \times 10^{2} \mathrm{~s}^{-1} \approx(3-4) \times 10^{-8} \omega_{\mathrm{pe}}$.

This value gives rise to the estimates of trapped electron number density

$n_{\mathrm{b}} \sim 10^{5} \mathrm{~cm}^{-3}$

because

$\gamma_{\max } \sim 10^{-2}\left(n_{\mathrm{b}} / n\right) \omega_{\mathrm{pe}}$.

The dimentionless energy density of the plasma waves is connected with the frequency and period of oscillations according to the equation (Zaitsev \& Stepanov 1983):

$w=\frac{W}{n T} \sim \frac{100}{f \tau} \approx 10^{-6}$. 
The brightness temperature of the radiation generated by the plasma mechanism at the second harmonic equals (Zaitsev \& Stepanov 1983):

$T_{\mathrm{b}}=a_{\omega} L_{\|}=\frac{(2 \pi)^{3}}{15 \sqrt{3}} \frac{c^{3} L_{\|}}{f_{\mathrm{pe}}^{2} V_{\mathrm{ph}}} \frac{w^{2}}{\zeta^{2}} n T$

where $a_{\omega}$ is the emission coefficient; $V_{\mathrm{ph}}$ is the transverse wave phase velocity (at $\left.f \approx 2 f_{\mathrm{UH}}\right) ; \zeta=\left(c / \omega_{\mathrm{pe}}\right)^{3}(\Delta k)^{3} \approx$ 30 for our case, see Eq. (31), relates to phase volume of plasma waves, and $L_{\|}$is the source size along the line of sight. On the other hand, the brightness temperature $T_{\mathrm{b}}$ is connected with the observed flux $F \approx 20$ sfu:

$T_{\mathrm{b}} \approx 1.4 \times 10^{26} \frac{F_{\mathrm{sfu}}}{f_{\mathrm{GHz}}^{2} L_{\perp}^{2}} \mathrm{~K}$

where $L_{\perp}(\mathrm{cm})$ is the source scale transverse to the line of sight. To apply these two equations we assume that the source sizes along and transverse to the line of sight are comparable in value:

$L_{\|} \sim L_{\perp}$.

This provides us with estimates of both brightness temperature

$T_{\mathrm{b}} \sim 5 \times 10^{12} \mathrm{~K}$

and the source scale

$L \sim 10^{7} \mathrm{~cm}$

in agreement with (16).

We should note that the frequency of radio emission, its flux and period of oscillations are very close to the pulsating event of November 17, 1991 (Fleishman et al. 1994a, 1994b). Nevertheless, here we found a larger value of the brightness temperature (44) and smaller source size (45) due to the smaller spectral bandwidth of the event under study.

\subsection{Mode shift due to group delay}

The time delay between $\mathrm{O}$ and $\mathrm{X}$ polarized waves originates naturally due to differences in group velocities of the waves in a magnetized plasma. The value of the magnetic field should be large enough for the effect of group delay to be observable. Let us evaluate plasma parameters of the region (along line of sight) providing most of the observed delay of $\sim 20 \mathrm{~ms}$.

In the Introduction we evaluated the group delay for an arbitrary frequency $\left(\omega^{2} \gg \omega_{\mathrm{pe}}^{2}\right)$. Here, we take into account the dependence of (twice) the upper-hybrid frequency on plasma frequency and gyrofrequency (18). If we introduce by definition the effective scale of inhomogeneity as

$H=\int_{0}^{\infty} \frac{\omega_{\mathrm{pe}}^{2}(z) \omega_{\mathrm{Be} \|}(z)}{\omega_{\mathrm{ps}}^{2} \omega_{\mathrm{Bs} \|}} \mathrm{d} z=\int_{0}^{\infty} \frac{n(z) B_{\|}(z)}{n_{\mathrm{s}} B_{\mathrm{s} \|}} \mathrm{d} z$

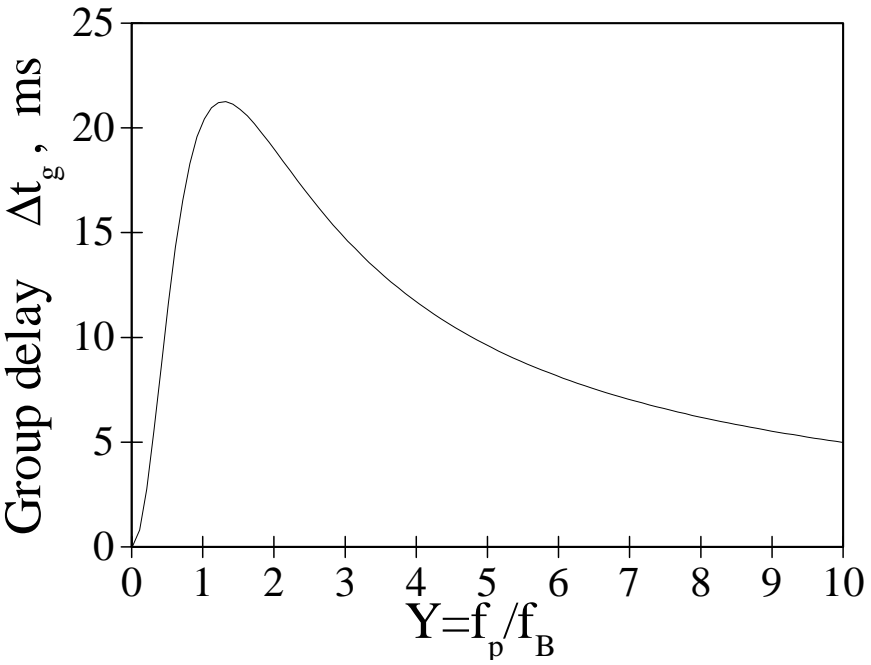

Fig. 11. Relative group delay between ordinary and extraordinary waves at frequency $f(Y)=2 f_{\mathrm{UH}}=2 \sqrt{f_{\mathrm{p}}^{2}+f_{\mathrm{B}}^{2}}$, which arises when propagating through a homogeneous magnetoactive plasma of the thickness $L=4.5 \times 10^{9} \mathrm{~cm}$ along the magnetic field, and its dependence on the ratio of plasma frequency to gyrofrequency. The curve is plotted with the use of exact expressions of the group velocities in cold magnetized plasma.

then the value of the group delay takes the form:

$\Delta t_{\mathrm{g}}=\frac{H}{4 c}\left(\frac{2 f_{\mathrm{UHs}}}{f}\right)^{3} \frac{1}{\left(1+Y_{\mathrm{s}}^{-2}\right) \sqrt{1+Y_{\mathrm{s}}^{2}}}$

where $f_{\mathrm{UHs}}, Y_{\mathrm{S}}$ are the respective averaged (over the source) values, $2 f_{\mathrm{UHs}} / f \approx 1$. Obviously, Eq. (47) reaches its maximum as a function of $Y$ (see Fig. 11) under condition (25). Now we can express the effective scale $H$ from Eq. (47):

$H \approx 4 c \Delta t_{\mathrm{g}}\left(1+Y_{\mathrm{s}}^{-2}\right) \sqrt{1+Y_{\mathrm{s}}^{2}}$.

Under the condition (25) and for $\Delta t_{\mathrm{g}} \approx 20 \mathrm{~ms}$ we find

$H \approx 5 \times 10^{9} \mathrm{~cm}$.

The respective Rotation Measure is

$R M=5.3 \times 10^{5} \mathrm{rad} / \mathrm{cm}^{2}$.

Benz \& Pianezzi (1997) calculated the dependence of group delay on plasma parameters. In particular, they found that the delay can be very (infinitely) large at frequencies closed to X-mode cut-off frequency. Our calculations do not display such extremely large delays because the emission frequency (19) is no longer an independent variable within the plasma mechanism and it cannot be too close to the X-mode cut-off frequency. This is the reason for the qualitative difference in behaviour of the group delay in Fig. 11, this paper, (the curve has a maximum), and in Fig. 5, Benz \& Pianezzi (1997) (the delay can become arbitrarily large). 


\section{Discussion}

Birefringence in magnetized plasma reveals itself particularly as the group delay effect. This paper discovers a good agreement between theoretical and observed frequency dependence of the delay, which proves reliably its group origin for the first time. The finding of a large group delay between two magnetoionic modes allows us to specify the microscopic emission mechanism providing the oscillating radiation. Namely, the nonlinear plasma mechanism operating close to twice upper-hybrid frequency provides pulsations of weakly polarized radio emission, while propagation effects lead to a considerable time delay between $L$ and $R$ radio emission, which results in a large and oscillating polarization degree.

The model developed here provides us with the following physical parameters.

For the source site:

- background plasma density: $n \approx 1.7 \times 10^{10} \mathrm{~cm}^{-3}$;

- magnetic field: $B \approx 290 \mathrm{G}$;

- kinetic electron temperature: $T \approx 3 \times 10^{6} \mathrm{~K}$;

- number density of fast electrons: $n_{\mathrm{b}} \sim 10^{5} \mathrm{~cm}^{-3}$;

- plasma wave growth rate: $\gamma_{\max } \sim 300 \mathrm{~s}^{-1}$;

- spectral bandwidth of generated plasma waves: $\Delta \omega / \omega \leq 3 \%$

- the wave number of generated plasma waves: $k d_{\mathrm{e}} \approx 0.1$; - the range of wave vectors of generated plasma waves: $c \Delta k / \omega_{\mathrm{pe}} \approx 3$ or $\Delta k d_{\mathrm{e}} \approx 0.1$

- the direction of maximal amplification of plasma waves: $\theta \approx 80^{\circ}$

- angular bandwidth of generated plasma waves: $\Delta \theta \approx 30^{\circ}$

- the level of plasma turbulence: $w=W / n T \approx 10^{-6}$;

- brightness temperature: $T_{\mathrm{b}} \approx 5 \times 10^{12} \mathrm{~K}$;

- linear scale of the source: $L \approx 100 \mathrm{~km}$.

For the delay site:

- rotation measure: $R M=5.3 \times 10^{5} \mathrm{rad} / \mathrm{cm}^{2}$;

- effective scale of inhomogeneity: $H \approx 5 \times 10^{9} \mathrm{~cm}$;

- variation of the group delay from lowest to highest frequency: $\delta t_{\mathrm{g}} \approx 1.5-2 \mathrm{~ms}$.

Therefore, the careful interpretation of radio data obtained with high temporal and spectral resolution provides very detailed diagnostics of the plasma both inside the emission source and along the line of sight. Particularly, the exited upper-hybrid waves are found to be anisotropically distributed, preferentially transverse to the magnetic field. Thus (as it is easy to see from the conservation laws), the radio waves are generated mainly along the magnetic field. This agrees well with the discovered earlier directivity of the radio pulsations: no flares connected with pulsations were observed between $70^{\circ}$ and $90^{\circ}$ (both east and west) away from the central meridian (Zlobec et al. 1987). We should note that the plasma parameters obtained here are consistent with the parameters derived by Huang \& Nakajima (2001) from the analysis of spike emission observed for the same event at a later phase.
Pulsations of solar radio emission with millisecond time scales have been recorded for many years by various radio observatories. Why has the effect of group delay between opposite polarizations of solar millisecond radio pulsations not been discovered before? The first reason is that millisecond pulsations are observed more frequently in the decimetric range, $f<1 \mathrm{GHz}$, higher in the solar corona, where the values of $Y_{\mathrm{s}}$ can be typically larger than the optimal value $Y_{\mathrm{s}}=1.4$, providing lower values of predicted group delays (see Fig. 11). On the other hand, the time resolution of most of the spectrometers used is of the order of $100 \mathrm{~ms}$ for the full band (Güdel \& Benz 1988; Allaart et al. 1990; Isliker \& Benz 1994), which is not enough to discover the effect. Single frequency observations of the pulsations in the $m-d m$ band revealed some events with a delay of about $30 \mathrm{~ms}$ that is close to the instrumental digitization rate (Zlobec et al. 1987). However, the origin of the delay was not established, particularly, the effect was not interpreted as the group delay.

Many pulsating events have been recorded at the Crimean Astrophysical Observatory at 2.5 and $2.85 \mathrm{GHz}$ with $10 \mathrm{~ms}$ resolution (Stepanov \& Yurovsky 1990; Yurovsky 1992a, 1992b). Unfortunately, the observations have been conducted in the total intensity only, which did not allow discovery of the effect as well.

The group delay effect has been studied for solar radio spikes. Benz \& Pianezzi (1997) found a rather small delay (of order of $100 \mu \mathrm{s}$ ) between different spike polarities. On the other hand, Zlobec \& Karlický (1998) and Wang et al. (2000) reported larger delays for solar radio spikes (2-8 ms), so the situation is not clear now and further study of the subject is strongly required.

Nevertheless, we can conclude that the group delay effect can be used as a very powerful tool for the interpretation of solar radio observations.

Acknowledgements. We would like to thank Drs. A. T. Altyntsev, A. S. Grebinsky, I. E. Kozhevatov and Y. Yan for fruitful discussions and an unknown referee for the comments that helped to improve the manuscript. The work was supported by Chinese Academy of Sciences and National NSF of China under grants No. 19773016, 19833050, and 49990451, as well as by the Russian Foundation for Basic Research under grants No. 99-02-16914, 00-02-16356, 01-02-16586, and Russian Federal Program "Astronomy".

\section{References}

Allaart, M. A. F., van Nieuwkoop, J., Slottje, C., \& Sonder, L. H. 1990, Sol. Phys., 130, 183

Aschwanden, M. J. 1987, Sol. Phys., 111, 113

Aschwanden, M. J., \& Benz, A. O. 1988, ApJ, 332, 466

Benz, A. O., \& Pianezzi, P. 1997, A\&A, 323, 250

Cordes, J. M., Weisberg, J. M., Frail, D. A., Spangler, S. R., \& Ryan, M. 1991, Nature, 354, 121

Fleishman, G. D., \& Melnikov, V. F. 1998, Uspekhi Fiz. Nauk, 168, 1265 (transl.: Physics - Uspekhi, 1998, 41(12), 1157)

Fleishman, G. D., Stepanov, A. V., \& Yurovsky, Yu. F. 1994a, Sol. Phys., 153, 403 
Fleishman, G. D., Stepanov, A. V., \& Yurovsky, Yu. F. 1994b, Space Sci. Rev., 68, 205

Fleishman, G. D., \& Korsakov, V. B. 1999, Proc. of 9th European Meeting on Solar Physics, Magnetic Fields and Solar Processes, Florence, Italy, 12-18 Sept. 1999 (ESA SP-448, Dec. 1999), 2, 803

Fu, Q. J., Qin, Z. H., Ji, H. R., \& Pei, L. B. 1995, Sol. Phys., 160, 97

Fu, Q., Ji, H., Liu, Y., et al. 2000, Astrophys. Rep., 36, 1

Güdel, M., \& Benz, A. O. 1988, A\&AS, 75, 243

Huang, G.-L., \& Nakajima, H. 2001, ApSS, submitted

Isliker, H., \& Benz, A. O. 1994, A\&AS, 104, 1451

Jiřička, K., Karlický, M., Mészárosová, H., \& Snižek, V. 2001, A\&A, 375, 243

Korsakov, V. B., \& Fleishman, G. D. 1998, Izv. Vuzov Radiofizika, 41, 46

Marple, Jr., S. L. 1987, Digital Spectral Analysis with Applications (Baltimore, Maryland)

Max J. 1981, Méthodes et techniques de traitement du signal et applications aux mesures physiques (Masson, Paris)
Pynzar', A. V. 1993, Astron. Zhurn., 70, 480

Slottje, C. 1981, Atlas of Fine Structures of Dynamic Spectra of Solar Type IVdm Bursts and Some Type II Radio Bursts, Dwingeloo

Solar Geophysical Data 1997, Part1, 640-641

Stepanov, A. V., \& Yurovsky, Yu. F. 1990, Pis'ma v Astron. Zh., 16, 247

Thompson, A. R., Moran, J. M., \& Svenson, G. W. 1990, Interferometry and Synthesis in Radio Astronomy (John Willey and Sons, Inc., N.Y.)

Wang, M., Fu, Q., Xie, R., \& Huang, G.-L. 2000, Astrophys. Rep., 36, 77

Yurovsky, Yu. F. 1991, Pis'ma v Astron. Zh., 17, 629

Yurovsky, Yu. F. 1992, Bull. Crimean Astrophys. Obs., 84, 182

Yurovsky, Yu. F. 1992, Bull. Crimean Astrophys. Obs., 85, 28 Zaitsev, V. V. 1971, Sol. Phys., 20, 95

Zaitsev, V. V., \& Stepanov, A. V. 1983, Sol. Phys., 88, 297

Zlobec, P., \& Karlický, M. 1998, Sol. Phys., 182, 477

Zlobec, P., Messerotti, M., Li, H.-W., Barry, M. B., \& Comari, M. 1987, Sol. Phys., 114, 375 